\title{
Brain's Computation on Near-death Experience and Dream
}

\author{
Qihua Chen
}

\author{
Nanjing Foreign Language School, Nanjing, 210008, China
}

ChenqihuaMaggie@outlook.com

\begin{abstract}
When big data are widely used in all walks of life, obsolete numerous information is processed by high-speed operation of the computer system: data collection, data cleaning, data mining, data presentation; thus, seemingly disorganized data rapidly become logical and interconnected. Numerous results not only can be seen but the trend can also be accurately predicted. The human brain is the most complicated system, known as the human body data center and computing center. A large number of neurons produce huge amounts of signals controlling the human body. So far, the exploration of the brain's computation on near-death experiences and dreams, because of the limitation due to the relatively small number of samples, is limited from the perspective of biology and dualism. Inspired by the principle of big data, I tried to analyze the origins of various illusions generated by the human brain from the perspective of big data.

The analysis is based on four steps of large data processing-data gathering, data cleaning, data mining, and data presentation-to represent how the human brain works when collecting signals, examining signals, forming the corresponding algorithm, and storing the algorithm.

This paper discusses the consistency of big data and how the brain work, which further illustrates that the experiences in the near-death experience and dreams are nothing but the results of the neuron activities. However, the results are different because of individual differences. What's more, through the research of these two specific conscious stages, this paper is dedicated to helping people who had negative emotions during near-death experiences or dreams by guiding them to objectively understand the processes.
\end{abstract}

Keywords: Brain's computation, near-death experience, dream

\section{INTRODUCTION}

Lewis Carroll said, "You are nothing but a pack of neurons." The emotions, sense of personal identity, and free will_everything people perceive is no more than the behavior of a vast assembly of nerve cells; in other words, the result of the computation of the human brain. We live in virtual reality which is created by the brain function.

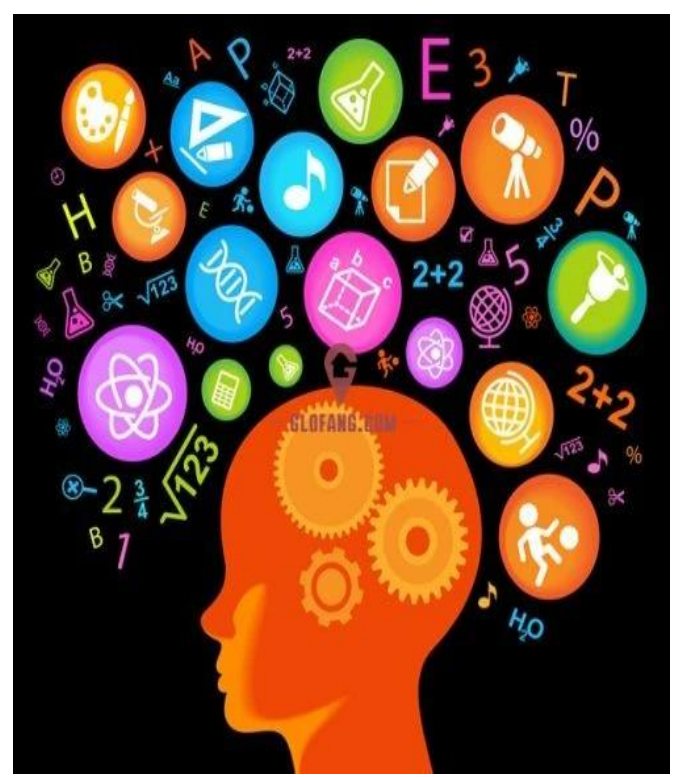

Figure 1 Computation of Human Brains 
Amazon defined big data as, "when your data sets become so large and diverse that you have to start innovating around to collect, store, process and analyze data." The formation of big data includes four stages: data collection, data cleaning, data analysis, and data presentation. Human brains are like computers and the way human brains use to deal with various information is based on the principle of big data. While people calmly serve our thoughts, inanimate computer processors and hard disks can be paralleled to thinking dimensions and the spiritual world: Human brain captures the energy senses, composed of vision, hearing, touch, and chemical senses including taste and olfaction, which is equivalent to the receptors inside the computers when gathering information. Those senses form the "metadata", the data describing the properties of the object, of our computation. This shows the value of big data: for the unstructured, unquantized objects, structured metadata can be used for quick calculations and judgments. Then, due to the top-down and bottom-up processing, human brains classify the information and give responses to the same set of stimuli next time. For example, after the human brain repetitively receives the element group consisting of grass, black-and-white fur, and main source of meat, and then learns that it is linked with the description of cows, the brain can form the memories in the brain's cortex. Later, every time they hear the same element group, they inevitably associate it with cows, which is consistent with the Gestalt Rule that people perceive images as groups instead of isolated elements. Computers, on the other hand, for instance, when persistently receive the typing of the word "abbreviate", the next time when it perceives the typing of "abb" (the first three letters of the "abbreviate"), it can reasonably infer the typing result of "abbreviate" and this response is stored in the disks [1].

The interesting question is, how does the human brain function at several unusual and critical stages, including near-death experience (NDE) and dreams. Based on the numerous previous studies on the analysis of near-death experience and dreams, we get the opportunity to take a closer look at those subjects and learn how those processes are linked to the brain's computation. In this essay, we are going to summarize how the human brain works as a computer, generates virtual reality by investigating the process of near-death experience, dreams as exemplifications. Analyzing those special physiological stages shed light on the further illustrations of computation on human brains.

\section{METHOD}

Due to the limitation of laboratory conditions, we fail to conduct any experiments. Nevertheless, we are determined to use the qualitative analysis of the numerous previous researches to gain sights of the computation of the human brain and the generation of virtual reality. Our essay will use the descriptive approach to explain the principles and theories with the collection of the secondary sources of peer-reviewed journal articles that are available on the online library of the University of Southern California, University of Edinburgh, and Web of Science. The query words are limited to Brain's Virtual Reality, Near-death Experience, Dream. By reading these references, we better concentrate on the brain's function during these unusual stages [2].

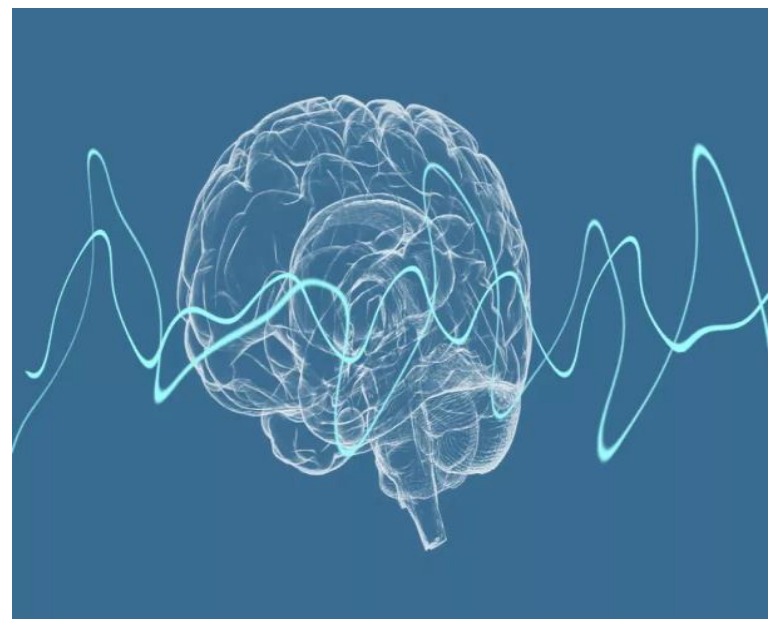

Figure 2 Spiritual Sources of Consciousness

\section{FINDINGS}

\subsection{Brain's Computation during the Near-death Experience}

Near-death experiences are subjective experiences happening during life-threatening conditions and involve reported memories of impressions during an unusual state of consciousness, consisting of several elements, such as an out-of-body experience, consciously returning to their body, seeing a tunnel, and a life review. Furthermore, as the NDEs can be divided as positive or negative ones based on the contents they undergo, a greater proportion of NDEs is positive. However, these phenomena can largely be explained by the computation of the brain. The fact that some people experience NDEs while others do not during the life-threatening stage is due to the different threshold of triggering or the previous data collection. In addition, the reason for the opposite or very divergent experiences during NDEs is that the "data cleaning" stage of the human brain is quite different among individuals. Consequently, "data presentation" is widely different according to the recollection of what happened during NDEs.

Generally, roles are divided into two types: bystanders and highly-involved participants (or even dominant ones). In NDEs, the self is mostly the identity of the bystander: either the self is separated from the physical body and watching what is happening around the body - the patients feel like taking off their bodies as old 
coats — or it is immersed in nihility, passively falling into a kind of illusion. The perception of viewing their bodies from a veridical angle above the lifeless body is called "out-of-body" experiences. According to a recent study of 93 near-death reports on out-of-body experiences, $90 \%$ were found to be completely precise, $8 \%$ contained some minor errors, and $2 \%$ were completely erroneous. Scientists suggest that the precision of the out-of-body experiences is based on memories stored in the human brain. This result suggested that the out-of-body experiences cannot be a mere hallucination or a delusion. Instead, it is based on "reality" created the computation of human brain. Also, some patients report they later consciously return to their physical bodies, usually from the head, which created a very oppressive feeling, and regain the consciousness of "being locked in their damaged bodies". From this point of view, though the role of the self in the NDE is passive, the experience is under the control of brain function [3].

The positive emotions experienced in NDEs are due to the endorphin that is released under stress, knowing to block the pain and create a feeling of well-being. What's more, cortical disinhibition associated with anoxia has been considered responsible for the perception of a tunnel and lights (Blackmore, 1996). The visual cortex consists of many cells devoted to the center of the visual field and few to the periphery. Therefore random excitation may produce the effect of bright light in the center fading out toward darkness, creating a sort of tunnel effect.

All in all, the continuous influence of self in NDE is deeply involved in the whole process. According to a recent study (van Lommel and colleagues, 2001), after several days of resuscitation, the patients recollected the memories of the unconsciousness: $18 \%$ reported that they remembered the time they were diagnosed as clinical death. In particular, $12 \%$ report a core experience. Based on the data, the brain function is still active since a much larger proportion of NDE experiencers maintains a clear memory [4].

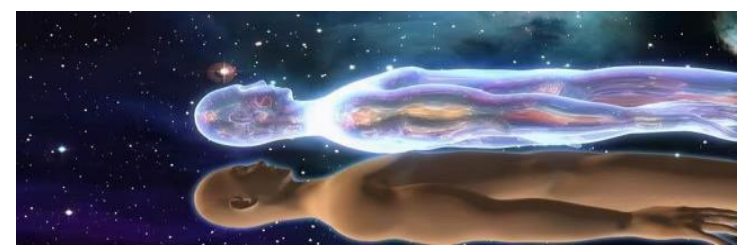

Figure 3 Near-death Experiences

Contrary to the doctrine of dualism, near-death experiences are not that mysterious and no more than the computation of the human brain. The imagination of the self is limited by the cognitive ability of the individual. The imagination of this manifestation stage is derived from a computation after data collection, data cleaning, and data mining of the real world. This computation is under a specific trigger mechanism. The corresponding data are presented. From the current statistical results, the scenes generated by the near-death experiences can be roughly divided into two categories: rescue scenes and miraculous roaming. Most individuals that go through the NDES are in the adult stage, which is also consistent with the fact that people of this age group begin to face and think about the problem of death, even if it is shallow thinking or instant thinking. This thinking process will generate certain data. It will eventually become a computation that is remembered by the brain. When different trigger mechanisms are activated, people will experience a preset scene: seeing a rescue or witnessing a miracle [5].

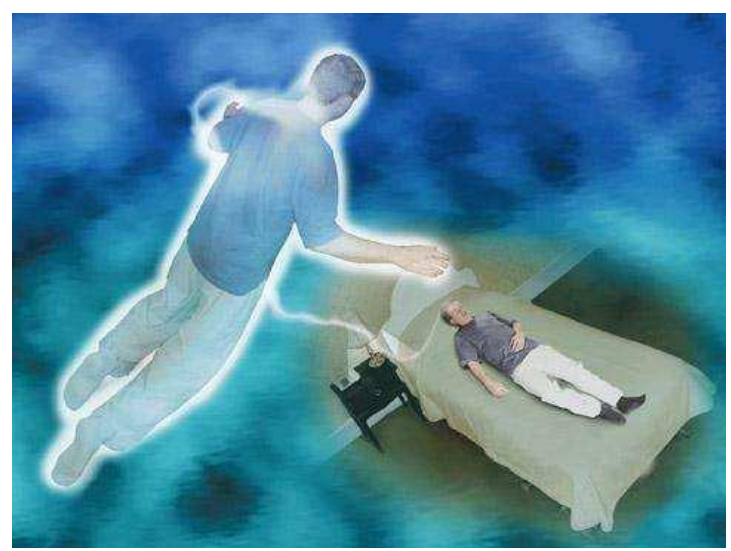

Figure 4 Out-of-body experiences

\subsection{Brain's Computation during Dreams}

Dreams are imagery information people experience when sleeping. The exploration of dream in Psychology has never ceased since the time of Sigmund Freud. According to Freud's psychodynamic theory, dreams are the expression of impulses and desires repressed during the daytime. Freud's successor, Carl Gustav Jung, believed that dreams reflected people's unconscious activities and contained many concepts of human archetypes. However, modern psychologists prefer to interpret dreams in terms of cognitive psychology: they are the product of a special pattern of brain activity arising from the neural activity generated by the memory of daily experiences during the day, and can therefore be viewed as a "drama" replayed in the brain by reassembling fragments of memory. Consequently, the reason why people have different contents in their dreams is that they collect various signals from the outside world during the day, which formed different algorithms stored in their brains. Thus, when they are presented in the form of dreams, they appear to diverge greatly. Moreover, some people frequently remember their dreams after waking up, while others fail to have clear memories about their dreams or are even unaware whether they were dreaming. Proponents of activation-synthesis theory view dreaming as a biological process. According to brain imaging, brains are highly active during rapid-eyemovement (REM) sleep. Human brains are very good at explaining things and dreams are the interpretations of 
what happened physiologically during REM sleep. Additionally, people can always remember what they dreamed after waking up from REM sleep. On the other hand, the information-processing theory points out that increased stress during the day can increase the intensity of the dream. Also, most people report that the content of the dream relates somehow to daily concerns. As the Chinese proverb said, "What you dream during the night reflects what you think during the day". Modern statistical results show that the contents of dreams have planning and associativity. The self can even have the predictability of dream content in the subconscious. Researches found"Day-Residue Effects": what happened in the previous day is often integrated into the dream (Josephine A. Henley-Einion and Mark T. Blagrove, 2014), and "Dream-Leg Effect": events 5-7 days ago is more likely to appear in our dreams than events 2-4 days or more ago (Strauch \& Meier, 1996).

However, the most intriguing category of dreaming is the lucid dream, which was first proposed in 1968 by philosopher Celia Green in her book Lucid Dreams, where people can realize that they are in the dream and thus, the self will drive the development of the dreams, such as making choices and creating dialogues in the dreams. J. Allan Hobson, a psychiatrist at Harvard medical school, found that lucid dreams have more GAMMA (40hz) brain activation patterns in the dorsolateral prefrontal cortex, although lucid dreams and regular dreams share the same input source (internal brain activity). In the book "The Interpretation of Dreams," Sigmund Freud described lucid dreaming as a "compulsive reappearance of the subconsciousness," referring specifically that when the brain has an obsession with something, it can convert a dream from an unconscious chaos into a semi-conscious state. Consequently, the role in the dream generally has a strong initiative character.

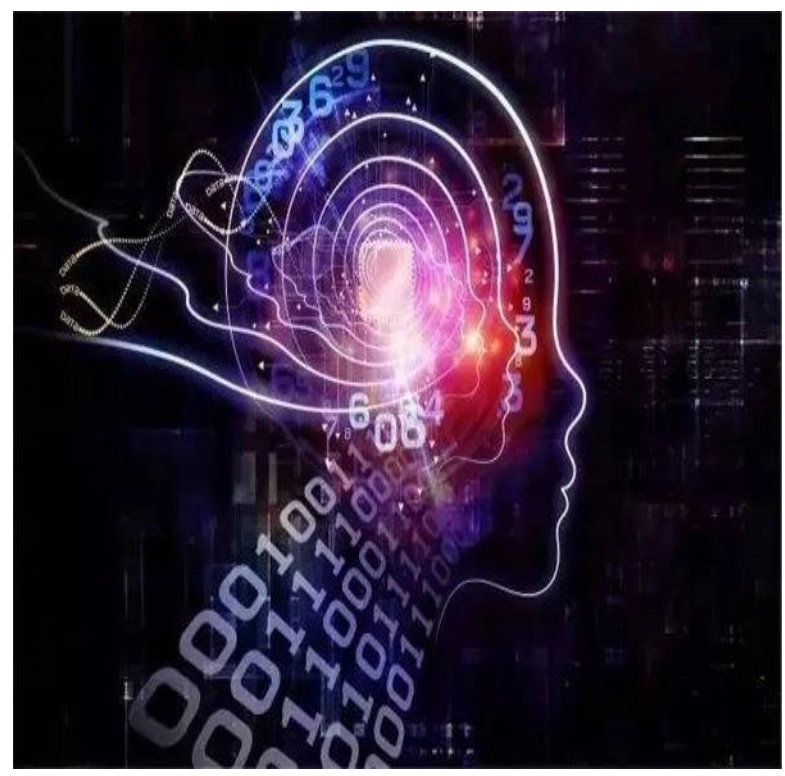

Figure 5 Brain's Computation in Dreams

\section{CONCLUSION}

From the perspective of developmental psychology, a human being start collecting data at birth. Informationprocessing theory suggests that the function of REM is to integrate the information processed during the day into our memories, so babies may need more REM sleep than adults because they process so much new information every day. Since the ability to learn is different among individuals, the stage of data cleaning is accordingly various. When it comes to data presentation and mining, the gap widens. Every single event reflects the computation and the frequency of the computing has a positive correlation with the frequency of the event. In near-death experiences, death is an event that only happens once throughout life while dreaming happens much more commonplace. Nevertheless, the understanding of the brain's function and computation during NDEs and dreaming is still limited due to the challenges of detecting brain activities and accessible devices. To advance the scientific understanding of these intriguing human experiences, many more studies are needed to fulfill the formation of explanatory hypotheses and the collection of high-quality empirical data to test these hypotheses.

\section{REFERENCES}

[1] Blanke, O., S. Ortigue, T. Landis, et al. 2002. Stimulating illusory own-body perceptions. The part of the brain that can induce out-of-body experiences has been located.Nature 419: 269-270.

[2] Blanke, O., Th. Landis, L. Spinelli, et al. 2004. Outof-body experience and autoscopy of neurological origin. Brain 127: 243-258.

[3] Blanke, O. \& Th. Metzinger. 2008 Full-body illusions and minimal phenomenal selfhood. Trends Cogn. Sci. 13: 7-13.

[4] De Ridder, D., K. Van Laere, P. Dupont, et al. 2007. Visualizing out-of-body experience in the brain. N. Engl. J. Med. 357: 1829-1933.

[5] Van Lommel, P. 2010. Consciousness Beyond Life. The Science of the Near-Death Experience. Harper Collins. New York. 